\title{
Stretchable Textile Based Supercapacitor with High Reliability
}

\author{
Seungmin Hyun, Tae Gwang Yun, Jeong Hwan Kim, Jae Sung Yoon, Chang-Su Woo \\ Korea Institute of Machinery and Materials \\ 156 Gajeongbuk-Ro, Daejeon, Republic of Korea \\ hyun@kimm.re.kr
}

\section{Extended Abstract}

There has been great interesting demand for flexible and wearable electronics due to huge potential applications. For advanced wearable electronics, flexible and stretchable energy storage system with high reliability is one of key parts. Supercapacitors are receiving much interest due to their high power density and long cycle life. Supercapacitors contain such components as electrodes, electrolytes, separators, and current collectors. To make flexible and stretchable supercapacitor, the proper selection of components including active materials and supporting substrate should be provided. Textile based supercapacitors are especially well-suited for wearable energy storage device applications due to their porous structure, which aids ion diffusion to enhance the specific capacitance compared to a flat polyester substrate. Textile supercapacitors also have the ability to maintain electrochemical performance under mechanical strain [1].

However, supercapacitors have a drawback of their low energy density compared to rechargeable Li ion batteries. To enhance the energy density, many researchers have studied nanostructured pseudocapacitor materials such as metal oxides [2]. However, large volume change of the metal oxide nanostructures during charge-discharge cycles cause delamination of the active materials, which decrease in electrochemical reliability. To prevent the delamination of nanostructured materials, thin layer of conductive polymer PEDOT:PSS is often coated on pseudocapacitor materials [3] to improve the electrochemical reliability. Although PEDOT;PSS serves as a conductive and adhesive layer, it does not have high capacity $(200 \mathrm{~F} / \mathrm{g}) \mathrm{It}$ is essential to use an alternative material serves as a conductive and adhesive layer while also enhancing capacitance.

In this study, we have developed highly reliable and stretchable supercapacitor using textile based electrode. Polypyrrole, $\mathrm{MnO} 2$ and Single Walled Nanotube (SWNT) coated textile electrodes for supercapacitors were prepared from pure cotton. The textile electrode was fabricated by two coating processes of SWNT dipping and electroplating of MnO2 and polypyrrole. The textile based supercapacitor was assembled using Poly ethylene oxide (PEO) based gel-type electrolyte. The polypyrrole-coated CNT textile supercapacitor with $\mathrm{MnO} 2$ nanoparticles and $\mathrm{MnO} 2$ coated CNT textile supercapacitor were evaluated for electrochemical performance and cyclic reliability. Polypyrrole-MnO2 coated CNT textile supercapacitor shows high electrochemical energy capacity up to $461 \mathrm{~F} / \mathrm{g}$ and high cyclic reliability up to $93.8 \%$ of energy capacity retention over 10,000 cycles. $\mathrm{MnO} 2$ coated CNT textile supercapacitor without polypyrrole thin layer retained 88.6\% normalized capacitance after 10,000 cycles. Polypyrrole coating increases 5.2\% improvement in the reliability. Energy density and power density of polypyrrole-MnO2 coated CNT supercapacitor were measured to be 31.1 $\mathrm{Wh} / \mathrm{kg}$ and $22.1 \mathrm{~kW} / \mathrm{kg}$, respectively. For the case of $\mathrm{MnO} 2$ coated CNT textile supercapacitor, the energy density was calculated as $14.2 \mathrm{Wh} / \mathrm{kg}$ and the power density were evaluated as $4.9 \mathrm{~kW} / \mathrm{kg}$. In situ measurement of the electrochemical performance change under mechanical strain of polypyrrole-MnO2 coated CNT supercapacitor shows $98.5 \%$ capacity retention at $21 \%$ tensile strain compared to the original discharge time. Cyclic bending tests under $13 \%$ strain were also performed while the electrochemical performance was measured. 750,000 bending cycles were tested on the supercapacitor. Cyclic voltammetry indicated $96.3 \%$ capacity retention. The fabricated flexible and stretchable energy storage device is suitable for wearable environments as demonstrated by mechanical tensile test and bending test results

\section{References}

[1] L. Liu, et. al., "Stretchable, porous, and conductive energy textiles," Nano Lett., vol. 10, pp. 708-714, 2010.

[2] C. Zhang, et. al., "Enhanced Electrochemical Performance of Hydrous RuO2/Mesoporous Carbon Nanocomposites via Nitrogen Doping,” ACS Appl. Mater. Interfaces, vol. 6, pp. 9751-9759, 2014. 
[3] G. Yu et. al., "Enhancing the supercapacitor performance of graphene/MnO2 nanostructured electrodes by Conductive Wrapping resonant circuit devices," Nano Lett., vol. 11, pp. 4438-4442, 2011. 